\title{
ON A RESULT OF JOHNSON ABOUT SCHUR MULTIPLIERS
}

\author{
by JAMES WIEGOLD
}

(Received 17 May, 1991)

The purpose of this short note is to give a new and shorter proof of the following theorem of Johnson [1], and to extend it somewhat.

THEOREM 1. Let $G$ be a finite non-cyclic p-group possessing a non-empty subset $X$ such that, for each $x$ in $X,\langle X \backslash\{x\}\rangle G^{\prime}$ is a complement for $\langle x\rangle$ in $G$. Then the Schur multiplier of $G$ is non-trivial.

In particular, the theorem applies to $p$-groups generated by elements of order $p$. Johnson's proof is homological in flavour, and I have always believed (see [2]) that one should be able to produce a quick, purely group-theoretical argument. In fact, we have:

THEOREM 2. Let $G$ be a finite group that can be expressed as a factor-group $G=F / R$, where $F$ is residually nilpotent, $R \neq 1$ and $R \leqslant F^{\prime}$. Then $M(G) \neq 1$. If in addition $F$ has trivial centre, there is an infinite sequence $G=G_{1}, G_{2}, \ldots$ of groups such that $G_{i+1}$ is $a$ non-trivial stem extension of $G_{i}$ for $i \geqslant 1$.

Proof. This is fairly standard, as is all the notation and terminology used here. Since $G \cong F /[R, F] / R /[R, F]$, it follows that $F /[R, F]$ has finite central factor-group, so that $F^{\prime} /[R, F]$ is finite and thus $F /[R, F]$ is finite since $R \leqslant F^{\prime}$. But then $R /[R, F]$ is an image of $M(G)$, and it is non-trivial since $F$ is residually nilpotent: if $R=[R, F]$, then $R \leqslant \gamma_{n}(F)$ for every $n$ (here, as usual, $\gamma_{n}(F)$ is the $n$-th term of the lower central series of $G$ ).

Now suppose that $F$ has trivial centre, and write $R_{i}$ for $[R, F, \ldots, F]$, with $i-1$ occurrences of $F$, so that $R_{1}=R$. Set $G_{i}=F / R_{i}$. Then $G_{i} \cong F / R_{i+1} / R_{i} / R_{i+1}$, and as before $R_{i} / R_{i+1}$ is an image of $M\left(G_{i}\right)$. Since $F / R_{i+1}=G_{i+1}$ and $R_{i} / R_{i+1} \leqslant G_{i+1}^{\prime}$, all we have to do is to show that $R_{i} \neq R_{i+1}$. If $R_{i}=R_{i+1}$, that is, $R_{i}=\left[R_{i}, F\right]$, we have $R_{i}=1$ since $F$ is residually nilpotent. Hence $i \geqslant 2$ since $R \neq 1$ by assumption, so that $\left[R_{i-1}, F\right]=1$ and thus $R_{i-1}=1$ since $F$ has trivial centre. This argument continues until we reach a contradiction to the fact that $R$ is non-trivial.

To recover Theorem 1 from Theorem 2, proceed like this. Suppose that the set $X$ figuring in Theorem 1 consists of elements $x_{1}, \ldots, x_{n}$ of orders precisely $p^{k_{1}}, p^{k_{2}}, \ldots, p^{k_{n}}$ respectively, and take for $F$ the free product of cyclic groups $\left\langle z_{1}\right\rangle, \ldots,\left\langle z_{n}\right\rangle$ of these same orders. Then $F$ is residually nilpotent, and $F$ and the kernel $R$ of the homomorphism extending the map $z_{1} \mapsto x_{1}, \ldots, z_{n} \mapsto x_{n}$ are readily seen to satisfy all the requirements of Theorem 2 .

\section{REFERENCES}

1. David L. Johnson, A property of finite $p$-groups with trivial multiplicator, Amer. J. Math. 98 (1976), 105-108.

2. James Wiegold, The Schur multiplier: an elementary approach, in Groups-St. Andrews, 1981, London Mathematical Society Lecture Note Series 71 pp. 137-154.

School of Mathematics, University of Wales College of Cardiff, CARDIFF CF2 4AG 